\title{
P-1150 Assessing the usage patterns of a diabetes education website available for Arabic speakers: insights and recommendations
}

\author{
Dari Alhuwail, PhD 1,2, Humoud Aljalahma, MHA², Heba Abduo, PGDipHeallnf, \\ BCPS $^{2}$, Ala'a Abu-Ghefreh, MSc, BC-ADM², Nadine Halawa, PharmD, BCPS ${ }^{3}$, \\ Basil Dawwas, BPharm²*, Naeema Alqabandi,
} MPharm², and Mohammed Alqersh, BCS ${ }^{2}$

* Presenting Author

1 College of Computing Sciences \& Engineering - Kuwait University, Kuwait

2 Dasman Diabetes Institute, Kuwait

3 Pharmacy Department - IWK Hospital, Halifax, Nova Scotia, Canada

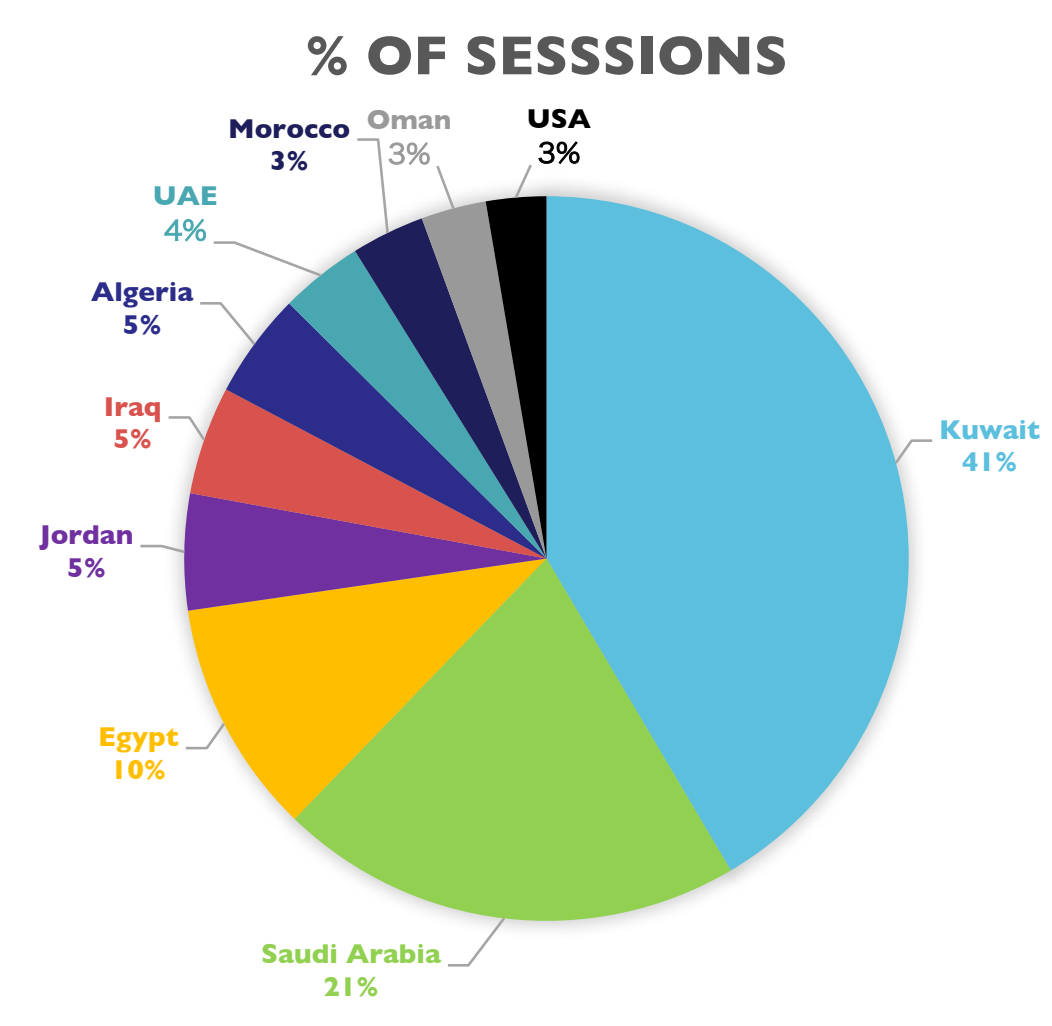

\section{BACKGROUND}

- Diabetes and its complications has become one of the top public health problems globally.

- More than 35 million people live with diabetes in the Middle East and North Africa (MENA) region.

- There is limited access to education and support resources tailored to the customs, culture, and language of diabetics living in the region. 2,3

- Evidence suggests that patients who are more actively involved in their own healthcare experience better outcomes and do not burden the healthcare system with high costs. ${ }^{4}$

- Health information technology (IT) has been showing potential to better engage patients in their own care.

- Unfortunately, accredited electronic resources to educate Arab diabetics are scarce. ${ }^{6}$

\section{AIMS}

i. Investigate the overall usage patterns and user profiles of an online diabetes website.

ii. Identify the website's components that were utilized most and least frequently.

iii. Offer recommendations to improve similar websites.

\section{METHODS}

- Diabetes Kuwait website (www.diabetes.org.kw) as part of the Diabetes Kuwait Resource Center initiative.

- Available in both the Arabic and English language

- Provides accredited and up-to-date information to the public targeting mainly patients with diabetes, their families/caregivers, and individuals who are at risk of developing diabetes.

- Information about diabetes in adult and pediatric populations, related disorders, treatment, and prevention strategies.

- Ability to submit medical questions as well as subscribe to a monthly newsletter.

- Contains a separate section for healthcare professionals, which is provided in English language only.

- Google Analytics: cumulative anonymous website usage data between January 2012 and March 2017 were analyzed.

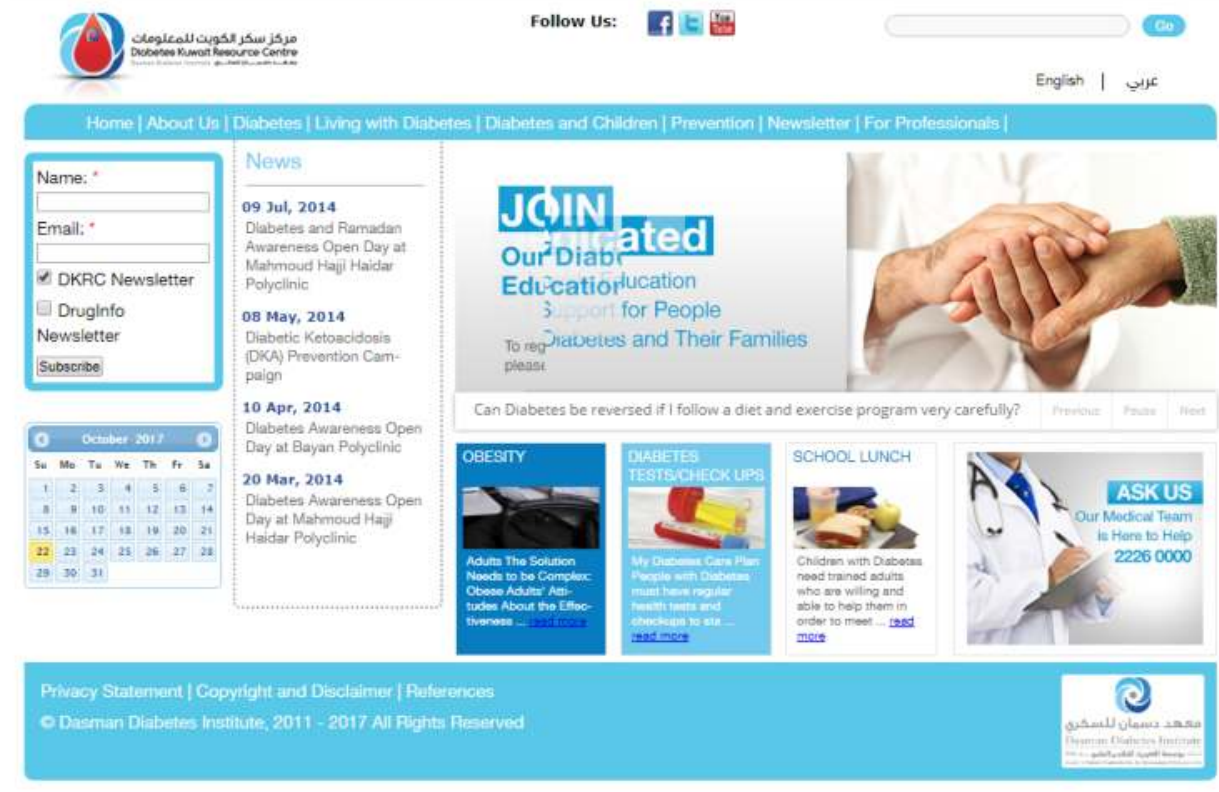

\section{RESULTS}

Reachability:

- Overall, 84,498 sessions with 72,587 visitors.

- More than two thirds of the site's visitors were from Arabicspeaking countries.

Access mode:

- Most visitors, 64,793 (76.7\%) used a search engine to access the site; only $13,439(16 \%)$ visitors accessed the site directly by entering the URL.

- The remaining visitors were referred from social media platforms, blogs, and similar websites. The referring social media platforms were mostly from Facebook (238) and Twitter (107).

Engagement:

- Only $10,325(12.2 \%)$ visitors spent more than one-minute on the website viewing more than two pages in each session.

- The majority, 74,173 (87.8\%), spent less than 30 seconds on the site and viewed one page.

- The landing pages viewed by the visitors included HbAlc test, diabetes treatments, myths about diabetes, pediatrics and diabetes, and healthy food recipes.

\section{Discussion}

- There is a need for a diabetes resource website for Arabic speakers.

- Majority of visitors used a smartphone to access the site

- Ensuring the diabetes education websites are mobile-friendly is critical to better engaging patients.

- Despite the great influence of social media on Arabic speakers, and its great potential in engaging diabetics, the current site did not fully leverage social media to engage with its visitors.

- Purposefully integrate healthcare organization social media channels with their electronic portfolio of educational resources. This is especially important considering the limited number of trusted and evidence-based social media channels that cater to the needs of Arab patients with diabetes.

- The information exchanged via websites and social media channels needs to be assessed and monitored for quality and reliability while maintaining the confidentiality and privacy. 